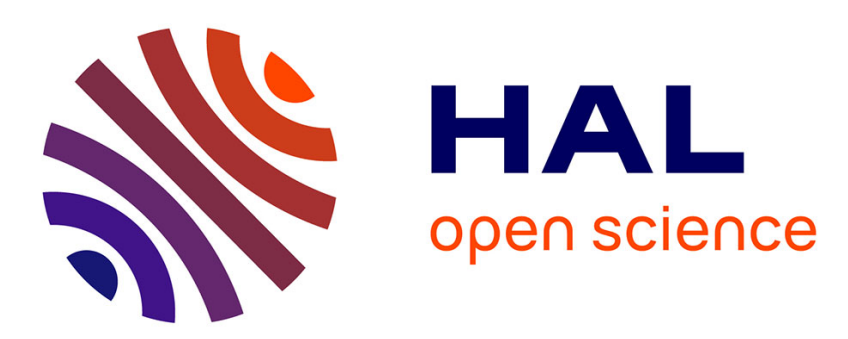

\title{
Rotation and contraction of native and regenerated cellulose fibers upon swelling and dissolution: the role of morphological and stress unbalances
}

Nicolas Le Moigne, Jérôme Bikard, Patrick Navard

\section{- To cite this version:}

Nicolas Le Moigne, Jérôme Bikard, Patrick Navard. Rotation and contraction of native and regenerated cellulose fibers upon swelling and dissolution: the role of morphological and stress unbalances. Cellulose, 2010, 17 (3), pp.Pages 507-519. 10.1007/s10570-009-9395-9 . hal-00509583

HAL Id: hal-00509583

https://hal-mines-paristech.archives-ouvertes.fr/hal-00509583

Submitted on 29 Apr 2011

HAL is a multi-disciplinary open access archive for the deposit and dissemination of scientific research documents, whether they are published or not. The documents may come from teaching and research institutions in France or abroad, or from public or private research centers.
L'archive ouverte pluridisciplinaire $\mathbf{H A L}$, est destinée au dépôt et à la diffusion de documents scientifiques de niveau recherche, publiés ou non, émanant des établissements d'enseignement et de recherche français ou étrangers, des laboratoires publics ou privés. 


\title{
Rotation and contraction of native and regenerated cellulose fibers upon swelling and dissolution: the role of morphological and stress unbalances
}

\author{
Nicolas Le Moigne, Jérôme Bikard \& P. Navard* \\ Mines ParisTech, CEMEF - Centre de Mise en Forme des Matériaux, CNRS UMR 7635, BP \\ 207, 1 rue Claude Daunesse, F-06904 Sophia Antipolis Cedex, France ; Member of the \\ European Polysaccharide Network of Excellence (EPNOE), www.epnoe.eu.
}

* Author for correspondence. E-mail: patrick.navard@mines-paristech.fr; phone: 33(0)493957466. Fax: 33(0)492389752

\begin{abstract}
Upon swelling and dissolution, native cellulose fibers such as cotton hairs or wood fibers are rotating and contracting. Regenerated cellulose fibers are only contracting, not rotating. Cotton hairs show two rotation mechanisms, a well known untwisting, not seen in wood fibers, due to the unwinding of the twists initially induced by the desiccation that occurs at the end of the growth, and a "microscopic rotation" that can also be slightly observed in wood fibers. In addition to these rotation mechanisms, cotton hairs and wood fibers show a rolling up of their primary wall that is due to the higher elongation of the external layers as compared to the internal layers arising during the elongation phase of the cell. Contraction originates from the fact that the cellulose chains are in an extended conformational state due to the spinning process for the regenerated fibers and to the bio-deposition process for native fibers. The contraction is related to the relaxation of the mean conformation of cellulose chains from an extended state to a more condensed state. Physical as well as mechanical modeling will support the experimental observations.
\end{abstract}

Keywords. Cellulose, rotation, contraction, stress, relaxation, conformation

\section{List of abbreviations}

$R_{c}=$ radius of the cylinders made by the rolled primary wall

$R_{\text {collars }}=$ radius of the collars

$L=$ unrolled length of the two primary wall cylinders

$e=$ thickness of the primary wall

$\Delta f_{x}=$ variation of the force per length unit along the $x$ axis

$\Delta f_{y}=$ variation of the force per length unit along the $y$ axis

$\Delta F_{x}=$ variation of the force along the $x$ axis

$\Delta F_{y}=$ variation of the force along the $y$ axis

$\Delta m_{x}=$ variation of the torque per length unit around the $x$ axis 
$\Delta m_{y}=$ variation of the torque per length unit around the $y$ axis

$\Delta M_{x}=$ variation of the torque around the $x$ axis

$\Delta M_{y}=$ variation of the torque around the $y$ axis

$\varepsilon_{x x}=$ deformation along the $x$ axis

$\mathcal{E}_{y y}=$ deformation along the $y$ axis

$\rho_{x}^{0}=$ initial radius of curvature along the $x$ axis

$\rho_{y}{ }^{0}=$ initial radius of curvature along the $y$ axis

$\rho_{x}=$ radius of curvature along the $x$ axis after rolling up of the primary wall

$\rho_{y}=$ radius of curvature along the $y$ axis after rolling up of the primary wall

$\mathrm{E}^{\prime \prime} ; v^{\prime \prime}=$ Young modulus and Poisson coefficient in the cellulose chains direction

$\mathrm{E}^{\perp} ; v^{\perp}=$ Young modulus and Poisson coefficient in the transverse direction to the cellulose chains

$L_{E}=$ length at the extended state

$L_{C}=$ length at the contracted state

$l=$ length of one monomer unit

$D P n=$ number average degree of polymerization

$M n=$ number average molar mass

$M w=$ weight average molar mass 


\section{Introduction}

When a material is composed of several parts having different physical properties, there is a possibility that upon changing physical, thermal or mechanical environment, this material will change its shape inhomogeneously. These changes are non-linear processes characterized by rotation or contraction movements due to morphological and stress unbalances. As an example, a simple bi-component structure (El-Sheikh et al. 1971; Paris 1992) composed of two materials with different expansion coefficients will bend upon changing the temperature. The same phenomenon occurs if a polymer part has a gradient of chain orientation and extension from one side to the other. This may occur during injection molding, a process characterized by a rapid cooling at the contact of the mold that freezes the chains in a well aligned manner with an extended mean conformation and by a slower cooling in the central part that allows the chains to be more disordered with a mean conformation closer to the one at equilibrium. When such a polymer part is heated, the molecular mobility allows the extended chains to move towards the equilibrium conformation, which decreases the dimension of the part in one direction, leading to a bending of the half-part (Isayev and Kwon 2009).

Cellulose fibers are good examples of complex composite materials made of various components and layers that are built upon biosynthesis (Klemm et al. 2002). It has been observed since long ago that non regular swelling occurs along native cellulose fibers. The most spectacular effect is the ballooning phenomenon where swelling takes place in some selected zones along the fiber (Nägeli 1864; Pennetier 1883; Flemming and Thaysen 1919, 1921; Hock 1950; Rollins and Tripp 1954; Tripp and Rollins 1952; Warwicker et al. 1966). More recently, Chanzy et al. (1983) and Cuissinat and Navard (2006a) showed that the solvent quality is a key parameter in the various swelling and dissolution mechanisms of cellulose fibers. The last authors described four main dissolution modes for cotton hairs and wood fibers as a function of the quality of the solvent in $\mathrm{N}$-methylmorpholine- $\mathrm{N}$-oxide (NMMO) - water with various amounts of water (the lower the amount of water is, the better the solvent is): fast dissolution by fragmentation below $17 \%$ water, large swelling by ballooning, followed by complete dissolution between 18 and $24 \%$ water, large swelling by ballooning, but with no complete dissolution between 25 and $35 \%$ water, low homogeneous swelling and no dissolution above $35 \%$ water. Similar mechanisms were also observed when using other solvents like $\mathrm{NaOH}$-water with or without additives (Cuissinat and Navard 2006b), ionic liquids (Cuissinat et al. 2008a) and other chemicals (Cuissinat 2006) for varied 
plant fibers (Cuissinat and Navard 2008) and for some cellulose derivatives that were prepared under heterogeneous conditions (Cuissinat et al. 2008b). In a recent paper (Le Moigne et al. 2008), the existence of a centripetal radial gradient in the dissolution capacity within cotton hairs was demonstrated. The sequence of dissolution was shown to be first the S2 wall, then the S1 wall and finally the primary wall meaning that the older was the cellulose deposition, the more difficult it is to dissolve it. These results were related to age-dependent structural re-organisations in the cell wall layers or to the presence of non-cellulosic polysaccharide networks in the outside walls. Aside solvent quality, the behaviour of native cellulose fibers upon swelling and dissolution is thus highly influence by the chemical and the morphological structure of the successive deposited layers.

The swelling of cellulose fibers is also accompanied by several macroscopic movements, as rotation or contraction of the fibers (Warwicker et al. 1966). A rotation effect, also called untwisting, is observed in cotton hairs immersed in a swelling agent. The magnitude of the rotation was found to increase with swelling agent of increasing quality, i.e. leading to larger swelling (Carra et al. 1962). This untwisting is supposed to be the reaction to the twisting (Warwicker et al. 1966) which arises from the removal of the liquid contained in the lumen, known as the dehydration or desiccation mechanism that occurs at the end of the growth (Hsieh 1999; Kassenbeck 1970; Warwicker et al. 1966). As quoted by Hsieh (1999), the twist frequency in the dried cotton hairs (3.9 to 6.5 per $\mathrm{mm}$ ) is higher than the $\mathrm{S}-\mathrm{Z}$ reversals in the cellulose layers (1 to 3 times per mm). Nevertheless, the author suggests that the twists must be related to the $S-Z$ reversals. The probability for the $S-Z$ reversals to coincide laterally in the concentric cellulose layers increases with fiber maturity and increasing secondary wall thickness. When a sufficient number of reversals coincides among the cellulose layers, stress leads to twists formation upon desiccation. The twists are thus supposed to be the regions where several reversals overlap among concentric layers. When put in a swelling agent, the stress released is sufficient to untwist cotton hairs. Contraction effects have been also reported. As an example, a well documented overview on swelling and contraction (also called shrinkage) of cotton hairs upon treatments in varying swelling agents is detailed in Warwicker et al. (1966).

All these studies show that rotation and contraction movements occur in cellulose fibers upon swelling and that they should have their origins in morphological and stress unbalances. However, no detailed description exists on the rotation and contraction mechanisms that occur upon the whole process of swelling and dissolution of cellulose fibers. The origins of these movements are also not or not fully discussed in the literature. The aim of this work is to 
study the rotation and contraction movements upon swelling and dissolution and their influence on swollen morphologies for three cellulose fiber sources, cotton hairs, wood pulp fibers and commercial regenerated fibers processed from a cellulose / NMMO solution (Lyocell process). The observed phenomena will be discussed in terms of morphological and stress unbalances induced upon biosynthesis (for natural fibers) and spinning (for regenerated fibers) processes and will be, whenever possible, physically and mechanically modeled.

\section{Materials and methods}

\section{Cellulose samples}

Three different cellulose samples were used: (i) cotton hairs, Gossypium barbadence with a fiber diameter of $15 \mu \mathrm{m}$ provided by INRA (France) and bleached cotton hairs with a similar fiber diameter and an average molar mass $M n=262900 \mathrm{~g} \cdot \mathrm{mol}^{-1}$ (degree of polymerization $D P n \approx 1630$ ), (ii) one sulphite spruce wood pulp fiber, BBUE with a fiber diameter of 20-30 $\mu \mathrm{m}$ and an intrinsic viscosity of $1700 \mathrm{ml} / \mathrm{g}$ (digested at a high kappa number meaning that the native cell wall structure is almost preserved) and one sulphite fir wood pulp fiber, VHV with a similar fiber diameter, an intrinsic viscosity of $1360 \mathrm{ml} / \mathrm{g}$ and an average molar mass $\mathrm{Mn}=$ 152600 g.mol ${ }^{-1}(D P n \approx 940)$ both provided by Borregaard (Norway) and (iii) regenerated cellulose fibers, Tencel ${ }^{\circledR}$, with a fiber diameter of $11 \mu \mathrm{m}$ and a $D P n \approx 340$ provided by Lenzing AG (Austria).

\section{Solvents}

In good quality solvents, the fast dissolution by fragmentation referenced to in the introduction does not allow analyzing rotation and contraction movements. Swelling agents induce only low rotation and contraction effects. We thus choose a mid-way, i.e. moderate quality solvents. The swelling and dissolution treatments were performed in solvents that are able to dissolve cellulose fibers after a large swelling, mixtures of anhydrous NMMO (provided by Sigma-Aldrich) and water prepared at $90^{\circ} \mathrm{C}$. The water content was varied from 20 to $23 \% \mathrm{w} / \mathrm{w}$ such as being able to fully dissolve (i) cotton hairs and wood fibers after the production of balloons and (ii) regenerated fibers after a large swelling.

\section{Experimental Protocol}

The experiments were performed by mixing the fibers and the solvent in a temperaturecontrolled container made of two glass plates separated with double-sided tape. The solvent, 
previously heated at $90^{\circ} \mathrm{C}$ and contained in a pipette, was introduced by capillary forces between the two plates maintained at the same temperature by a Linkam TMS 91 hot stage. Fibers were freely floating in the solvent and no agitation was applied to the mixture. The contraction ratios were determined by fixing the fibers at one end and by measuring their length before solvent introduction and after the large swelling observed prior to full dissolution. In the case of wood pulp, the short length of the fibers does not allow to fix it. In this case, the contraction was evaluated by considering the contraction between two collars as reference visual points (as will be described in the Results and discussion section, the collars correspond to zones between the balloons). To check the effect of preventing rotation (Figure $5 b$ ), cotton hairs were fixed under low tension at both ends as was previously achieved in (Le Moigne et al. 2009).

\section{Observation methods}

The swelling and dissolution of cellulose fibers were observed by optical microscopy in transmission mode with a Metallux 3 (Leitz). To obtain good images, the microscope was equipped with a high resolution numerical reflex camera $(3000 * 2000$ pixels) CANON D100 (Figure 1, 4, 5a, 6a) and a high resolution 3-CCD camera (1360*1024 pixels) JVC KY-F75U (Figure 5b, 6b, 7, 8). Fibers were also observed by scanning electron microscopy used in environmental mode with the following parameters: $15 \mathrm{kV}, 5.5$ mbar, relative humidity $30 \%$ (Figure $1 b, 3$ ). In this case, the dissolution process was stopped by a sudden addition of water and the fiber was extracted from the two glass plates and deposited on the sample holder for observations.

\section{Results and discussion}

\section{Detailed description of the first swelling and dissolution steps for native cellulose fibers.}

The classical explanation for the ballooning phenomenon in native cellulose fibers is that the swelling of the cellulose present in the secondary wall is causing the primary wall to extend and burst. According to Hock (1954): "the expanding swollen cellulose pushes its way through the tears in the primary wall, the latter rolls up in such a way as to form collars, rings or spirals which restrict the uniform expansion of the fiber" and form balloons.

As was recently demonstrated, the secondary S2 wall is the easiest to dissolve as compared to the external walls which contain larger amount of non-cellulosic components (Le Moigne 
et al. 2008). The solvent thus goes inside the fiber through the primary wall which is permeable to the solvent but not easy to dissolve and not extensible (Figure 1a). In moderate quality solvent, it was shown that the S2 wall dissolves by fragmentation (Le Moigne et al. 2008) and as a consequence, the S1 wall swells transversely. Optical observations show that the primary wall breaks (Figure $1 \mathrm{~b}$ ) in one or more places under the pressure of the $\mathrm{S} 1$ wall swelling and then rolls up. Finally, balloons are formed due to the large swelling of the S1 wall (Figure 1c). The primary wall that is cut and rolled up forms threads (seen as thin lines laid down along the balloon surface) and / or collars (regroupment of the rolled primary wall around the fiber diameter, preventing fibers to swell) as seen on Figure 1c. The selection between threads and collars is depending on the way the primary wall is broken, i.e. depends of the shape of the initial cut. When cut occurs on the whole circumference of the fiber, the primary wall rolls up along the fiber direction in the two opposite directions and forms only collars. If cut is more local and directed along the fiber axis, the primary wall rolls up perpendicularly to the fiber axis and forms one or more threads attached to two collars (Figure $1 \mathrm{~b}$ and 1c). The collars block the swelling of the fiber, forming what has been called the unswollen sections (actually a region between two balloons) in Cuissinat and Navard (2006a). When the S2 wall is fully dissolved, the swelling of the balloons reaches its maximum size (average swelling ratio from 5 to 5.5 for cotton hairs and BBUE wood pulp fibers). The balloon is thus formed of the dissolved $\mathrm{S} 2$ wall cellulose inside an undissolved membrane composed of the swollen S1 wall, surrounded by one or more threads of the primary wall and delimited by two collars (Figure 1c). As we demonstrated recently (Le Moigne et al. 2008), the $\mathrm{S} 1$ wall then the primary wall will slowly dissolve later.

\section{Rolling up of the primary wall}

\section{Morphological origins}

Upon ballooning of cotton hairs and wood pulp fibers, the primary wall could well break in many pieces falling apart and detaching from the secondary wall instead of rolling up on itself after breaking. To roll implies that there are frozen stresses inside the primary wall that are released after cutting. These frozen stresses should originate from a gradient of cellulose chain orientation and stretching from the outside to the inside of the primary wall, obviously not due to a gradient of cooling as was previously discussed for classical thermoplastic polymer processing, but related to the bio-deposition of the cellulose chains. Upon biosynthesis, the primary wall is built and at the same time elongated. The first layers of the 
primary wall are the ones being the most elongated in the fiber direction, while the subsequent layers are deposited on already elongated layers (Ryser 1999). This induces a much larger deformation of the first layer deposited than the layer deposited when the cell is at almost its maximum length size. The high elongation of the external layers is relaxed when the primary wall is broken, leading to its rolling up, as illustrated on Figure 1b and 1c. The rolling up occurs with the outside of the primary wall inside the roll, proving that it is the outside layers of the primary wall that are bearing the maximum stress, due to the fact that cellulose chains are more oriented and stretched than the inside layers.

As shown on Figure 1c, the threads created by the rolling up of the primary wall are composed of two small cylinders. This comes from the fact that the two lips of the cut primary wall roll up in opposite directions and meet at the other side of the fiber. When the primary wall rolls up along the fiber direction to form collars, same cylinders are observed at both extremities of the collars. The diameters of each of these cylinders vary from 2.2 to 2.7 $\mu \mathrm{m}$ for cotton hairs and wood fibers. Considering the thickness of the primary wall $e$ which is in the range 0.1 to $0.4 \mu \mathrm{m}$ according to Klemm et al. (1998) Ryser (1999) and Warwicker et al. (1966), we can calculate the unrolled length $L$ if we would unroll the two cylinders. This length $L$ can be calculated as follows:

$$
\begin{gathered}
\text { Surface of the cylinders }=2 \times \pi \times\left(R_{c}\right)^{2}=L \times e \\
\text { giving, } L=2 \times \frac{\pi \times\left(R_{c}\right)^{2}}{e}
\end{gathered}
$$

The unrolled length $L$ of the rolled primary wall can be compared to the initial perimeter of the fibers before swelling. As can be seen on Figure 2, the calculated unrolled length is in the proper order of magnitude for initial fiber diameter between 10 to $30 \mu \mathrm{m}$ if the thickness of the primary wall is in the range of $0.1 \mu \mathrm{m}$ to $0.3 \mu \mathrm{m}$.

\section{Mechanical analysis}

The magnitude of rolling is linked to the morphological and stress unbalances between the two sides of the primary wall and should give an estimation of the level of stress released after breaking. The mechanical analysis of such a process was introduced for metal parts by Treuting and Read (1951), and applied to polymers by many authors for injected pieces (Baaijens 1991; Giroud and Vincent 2004) or asymmetrical fibers (Hensen 1989; Matsuo 1977). In these studies, the magnitude of bending was low and thus modeled by low deformation theories. In the present study, the rotation is very high since the primary wall 
totally rolls up on itself. The scheme of the rolling up of the primary wall when considering a breaking of the primary wall on the whole circumference (case of collars formation) is given on Figure 3. However, the system can be still interpreted with small deformation theories when considering the deformation of a representative elementary volume (Figure 3), the macroscopic deformation being the sum of the small bending of each representative elementary volume. For simplification reason, we consider the primary wall as a bi-layered structure (delimited by a dash line on Figure 3) with the outside layer made of cellulose chains oriented along the fiber axis $(x)$ and the inside layer made of cellulose chains oriented transversally to the fiber axis $(y)$. In reality, cellulose chains progressively switch from the fiber axis direction to the perpendicular direction.

The variations of the forces per unit length within the thickness of the primary wall for a representative elementary volume are:

$$
\left(\begin{array}{c}
\Delta f_{x} \\
\Delta f_{y}
\end{array}\right)=\left(\begin{array}{l}
\int_{-e / 2}^{e / 2} \sigma_{x x} d z \\
\int_{-e / 2}^{e / 2} \sigma_{y y} d z
\end{array}\right)=\left(\begin{array}{l}
\int_{-e / 2}^{0} \frac{E^{\perp}}{1-v^{\perp} v^{\prime /}}\left[\varepsilon_{x x}+v^{\perp} \varepsilon_{y y}\right] d z+\int_{0}^{e / 2} \frac{E^{\prime \prime}}{1-v^{\perp} v^{\prime \prime}}\left[\varepsilon_{x x}+v^{\prime \prime} \varepsilon_{y y}\right] d z \\
\int_{-e / 2}^{0} \frac{E^{\prime \prime}}{1-v^{\perp} v^{\prime /}}\left[\varepsilon_{y y}+v^{\prime \prime} \varepsilon_{x x}\right] d z+\int_{0}^{e / 2} \frac{E^{\perp}}{1-v^{\perp} v^{\prime \prime}}\left[\varepsilon_{y y}+v^{\perp} \varepsilon_{x x}\right] d z
\end{array}\right)
$$

The deformations along the $x$ direction $\left(\varepsilon_{x x}\right)$ and the $y$ direction $\left(\varepsilon_{y y}\right)$ have different origins. $\varepsilon_{x x}$ is the consequence of the rolling up of the primary wall (flexion mechanisms) and $\varepsilon_{y y}$ is due to the fact that, when the primary wall rolls up, the thickness of the cylinders created is larger than the initial thickness of the primary wall (traction mechanisms). In small deformations we can write that:

$$
\left(\begin{array}{l}
\varepsilon_{x x} \\
\varepsilon_{y y}
\end{array}\right)=\left(\begin{array}{c}
z\left[\frac{1}{\rho_{x}}-\frac{1}{\rho_{x}{ }^{0}}\right] \\
\frac{\rho_{y}-\rho_{y}{ }^{0}}{\rho_{y}{ }^{0}}=\frac{-\rho_{x}}{\rho_{y}{ }^{0}}
\end{array}\right)
$$

According to the geometry of the primary wall before its breaking: $\rho_{x}=-R_{c} ; \rho_{x}^{0}=\infty ; \rho_{y}^{0}=$ $R_{\text {collars }}$, we can write that after the breaking and the rolling up of the primary wall, the deformations of the representative elementary volume along $x$ and $y$ axes reduce in:

$$
\left(\begin{array}{c}
\varepsilon_{x x} \\
\varepsilon_{y y}
\end{array}\right)=\left(\begin{array}{c}
-\frac{z}{R_{c}} \\
\frac{R_{c}}{R_{\text {collars }}}
\end{array}\right)
$$


such that the variations of the forces per unit length within the representative elementary volume along $x$ and $y$ axis become:

$$
\left(\begin{array}{c}
\Delta f_{x} \\
\Delta f_{y}
\end{array}\right)=\left(\begin{array}{l}
-\left[\frac{e^{2}}{8 R_{c}\left(1-v^{\perp} v^{\prime \prime}\right)}\right]\left[E^{\prime \prime}-E^{\perp}\right]+\left[\frac{e R_{c}}{2 R_{\text {collars }}\left(1-v^{\perp} v^{\prime \prime}\right)}\right]\left[v^{\prime \prime} E^{\prime \prime}+v^{\perp} E^{\perp}\right] \\
{\left[\frac{e^{2}}{8 R_{c}\left(1-v^{\perp} v^{\prime \prime}\right)}\right]\left[v^{\prime \prime} E^{\prime \prime}-v^{\perp} E^{\perp}\right]+\left[\frac{e R_{c}}{2 R_{\text {collars }}\left(1-v^{\perp} v^{\prime \prime}\right)}\right]\left[E^{\prime \prime}+E^{\perp}\right]}
\end{array}\right)
$$

If we consider : $E^{\prime \prime}=220 \mathrm{GPa}$ and $E^{\perp}=15 \mathrm{GPa}$ (Diddens et al. 2008); $e=0.3 \mu \mathrm{m} ; R_{c}=1.3$ $\mu \mathrm{m} ; v^{\prime \prime}=v^{\perp}=v$ and $v=0.49$ (classical value for semi-crystalline polymers) $; R_{\text {collars }}=$ $15 \mu \mathrm{m}$. The variations of forces per length unit in $x$ and $y$ directions are:

$$
\left(\begin{array}{c}
\Delta f_{x} \\
\Delta f_{y}
\end{array}\right)=\left(\begin{array}{l}
-364 \mathrm{~N} \cdot \mathrm{m}^{-1} \\
5164 \mathrm{~N} \cdot \mathrm{m}^{-1}
\end{array}\right)
$$

According to the perimeter of the fiber, the total forces released during the rolling up are: $\left(\begin{array}{l}\Delta F_{x} \\ \Delta F_{y}\end{array}\right)=\left(\begin{array}{c}-0.036 \mathrm{~N} \\ 0.487 \mathrm{~N}\end{array}\right)$

$\Delta F_{x}$ is negative because it corresponds to the relaxation of the stress that was stored during the elongation phase of the primary wall. $\Delta F_{y}$ is positive because it is generated by the increase of thickness of the cylinders created upon the rolling up. It has to be emphasized that the Young modulus considered for the calculation should be lower since the solvent should have already broken a part of the hydrogen bonds within the primary wall, even if it fully dissolves at a late stage. As an example, if the values of modulus are ten times lower $\left(E^{\prime \prime}=\right.$ $22 \mathrm{GPa}$ and $\left.E^{\perp}=1.5 \mathrm{GPa}\right)$, it gives:

$$
\left(\begin{array}{l}
\Delta F_{x} \\
\Delta F_{y}
\end{array}\right)=\left(\begin{array}{c}
-0.004 \mathrm{~N} \\
0.049 \mathrm{~N}
\end{array}\right)
$$

These values can be compared to the breaking force of the primary wall which is about $0.01 \mathrm{~N}$ according to Hsieh (1999). The simplifications done in the mechanical analysis of the rolling up of the primary wall are giving results close to the independently determined breaking force. It is interesting to see that $\Delta F_{y}$ is higher than $\Delta F_{x}$ which is normally the case in the mechanics of thin walls. Also, $\Delta F_{y}$ seems to be higher than the breaking force which means that the $\Delta F_{y}$ magnitude generated should lead to the breaking of the primary wall along the fiber axis. It is in agreement with the observations of thread formation previously described.

The torque around $x$ and $y$ axis upon the rolling up of the primary wall can be also calculated. The variations of the torques per length unit length for a representative elementary volume are: 


$$
\left(\begin{array}{l}
\Delta m_{x} \\
\Delta m_{y}
\end{array}\right)=\left(\begin{array}{c}
-\left[\frac{e^{3}}{24 R_{c}\left(1-v^{\perp} v^{\prime \prime}\right)}\right]\left[E^{\prime \prime}+E^{\perp}\right]+\left[\frac{e^{2} R_{c}}{8 R_{\text {collars }}\left(1-v^{\perp} v^{\prime \prime}\right)}\right]\left[v^{\prime \prime} E^{\prime \prime}-v^{\perp} E^{\perp}\right] \\
-\left[\frac{e^{3}}{24 R_{c}\left(1-v^{\perp} v^{\prime \prime}\right)}\right]\left[v^{\prime \prime} E^{\prime \prime}+v^{\perp} E^{\perp}\right]+\left[\frac{e^{2} R_{c}}{8 R_{\text {collars }}\left(1-v^{\perp} v^{\prime \prime}\right)}\right]\left[-E^{\prime \prime}+E^{\perp}\right]
\end{array}\right)
$$

giving total torques, $\left(\begin{array}{l}\Delta M_{x} \\ \Delta M_{y}\end{array}\right)=\left(\begin{array}{c}-1.31 \mathrm{E}-9 \mathrm{~N} . \mathrm{m} \\ -3.71 \mathrm{E}-9 \mathrm{~N} . \mathrm{m}\end{array}\right)$ with $E^{\prime \prime}=22 \mathrm{GPa}$ and $E^{\perp}=1.5 \mathrm{GPa}$.

$\Delta M_{y}$ is the driver of the rolling up that occurs around the $y$ axis. $\Delta M_{x}$ is due to the increase of the radius of curvature of the cylinders from the beginning to the end of the rolling up that creates a torque around the $x$ axis.

\section{Rotation in native and regenerated cellulose fibers}

\section{Cotton hairs}

Upon swelling and dissolution, cotton hairs are also showing two rotations. One is a "macroscopic rotation" of the hairs due to an untwisting upon swelling as was previously discussed in the Introduction section. Twists of cotton hairs in dried state are shown in Figure 4.

A second rotation, that we will call the "microscopic rotation", occurs when the cotton hairs are swelling and dissolving. This rotation induces the formation of helices around the balloons from the rolled threads (Figure 5). In this case the rotation is about $180^{\circ}$. Depending of the way the primary wall broke (leading to one or more rolled threads), one or more helices can be observed as shown in Figure 5. The "microscopic rotation" is clearly visible when comparing cotton hairs which were freely floating in the solvent and thus swelling by ballooning, and cotton hairs which were fixed at both ends to prevent the rotation and swelling homogeneously. In the first case, the microfibril arrangements of the S1 wall (seen as thin lines) within the membrane are more or less aligned along the fiber axis after rotation (Figure 6b). On the other hand, when preventing the rotation, the microfibrils are oriented helically towards the fiber axis (Figure 6a) closest to the orientation that exists at the end of the growth, which is about 20 to $30^{\circ}$ according to Hsieh (1999) and Warwicker et al. (1966).

\section{Wood pulp fibers}

There is no "macroscopic rotation" for BBUE wood pulp fibers. The "microscopic rotation" phenomenon is slightly seen but not as clear and well defined as in cotton hairs. The primary wall roll threads mostly stay at the edge of the balloons as shown on Figure $7 \mathrm{a}$, 
implying that no microscopic rotation occured. However, some helices were sometimes observed with BBUE wood pulp fibers. This was attributed to the way the primary wall cut during the first stage of ballooning which can creates helices without the necessity to perform a rotational movement. In fact, if the cutting of the primary wall occurs at two close but opposite places towards the diameter of the fiber, the rolling up of the primary wall creates helices, or at least rings, without rotation (see Figure 8).

\section{Regenerated cellulose fibers}

Regenerated cellulose fibers do not show any rotation and as was reported in a recent work (Chaudemanche and Navard 2009), the morphology before total dissolution is a large homogeneous swelling (Figure 7b). Regenerated cellulose fibers have a simple, well oriented morphology along the fiber axis without the complex wall structure of native cellulose fibers even if a skin-core morphology exists due to the sudden contact of the spun solution in the regenerated bath (Abu-Rous et al. 2006; Jianchin et al. 1999).

\section{Morphological origins of the "microscopic rotation"}

The "microscopic rotation" is the one that is observed mostly for cotton hairs and slightly for BBUE wood pulp fibers during the large swelling and dissolution of the S2 wall. There is a rotation of the fibers when they are swelling because frozen stresses are released. The fact that regenerated fibers are not showing any rotation suggests that the origin of this "microscopic rotation" lies in the deposition structure and kinetics of the S1 and/or S2 layers of cotton and wood cells. A first morphological parameter to recall is the angles the cellulose microfibrils are making towards the wood fiber or cotton hair axis. Cellulose microfibrils in wood fiber have orientation angle of about 50-70 (Roelofsen 1959) or 70-90 (Brändström et al. 2003) in $S 1$ wall and $0-30^{\circ}$ in $S 2$ wall (Sahlberg et al. 1997). In cotton hairs, these angles are $20-30^{\circ}$ and $35-45^{\circ}$ for $S 1$ and S2 wall, respectively (Warwicker et al. 1966). The cellulose chains are thus deposited at about these angles upon the biosynthesis and the Cellulose Synthase Complexes (CSCs) are moving along the wall with the same angles, as was recently visualized (Paredez et al. 2006). When performing this deposition, there should be no associated stress unbalances and there should be no stress released during swelling since the equilibrium state is this helical deposition. There is clearly something missing in the understanding of fiber deposition in order to explain this microscopic rotation. 


\section{Contraction in native and regenerated cellulose fibers}

In addition to the rolling up of the primary wall and the rotation phenomena described above, a contraction of the fibers is also observed upon swelling and dissolution. We recently reported (Le Moigne et al. 2009) that the contraction forces occurring during swelling and dissolution are so high that they can break a fiber when it is maintained at its two ends. As shown on Figure 9, ballooned VHV wood pulp fibers undergo large contraction with contraction ratio of about 1.7. Similar contraction ratios were also measured for cotton hairs (about 1.8 for bleached cotton) and regenerated fibers (about 2). The contraction ratio is defined as [initial length of the fiber] / [length of the contracted fiber].

Such large contractions are clearly related to the mechanism of cellulose chains deposition in cell walls for native fibers and to the spinning process for regenerated fibers. In both cases, cellulose chains are well aligned, extended and strongly oriented. In the case of regenerated fibers, the cellulose solution dope is very viscous and passes through a spinneret where shear forces orient the cellulose chains in the spinning direction. The resulting cellulose fibers are then strongly elongated in an air gap before being suddenly coagulated in a water bath. Cellulose chains are frozen in a strongly extended conformation, out of equilibrium. The result is the well-known highly oriented morphology of regenerated cellulose fibers where both the crystalline and non-crystalline phases have their cellulose chains strongly oriented in the fiber direction (Lenz et al. 1994; Schurz 1994). As suggested by Bowling et al. (2001), cellulose chains in cotton hairs or wood fibers are also in an extended conformational state, out of equilibrium. Here, the reason is the bio-deposition mechanism, where the Cellulose Synthase Systems extrude cellulose chains that are condensing into crystalline microfibrils before they can totally relax, thus maintaining the chains in an extended conformational state. As postulated by O'Sullivan (1997), even chains in non-crystalline areas probably still possess a degree of order.

Upon partial dissolution or swelling, molecular mobility allows cellulose chains belonging to the non-crystalline phase (often called amorphous phase) to return closer to their equilibrium conformation, with a much smaller end-to-end distance. The equilibrium end-toend distance value depends on the swelling or dissolving agent. The strong network of hydrogen bonds among all chains (Klemm et al. 1998; Krässig 1993) brings the whole cellulose fiber to contract due to this return to conformational equilibrium of individual chains. This is most probably a non linear process, some chains being able to contract without participating to the contraction of the whole fiber. 
The values of contraction of cotton hairs, wood pulp fibers and regenerated fibers can be compared to those of flexible polymer chains and molecularly dissolved cellulose chains as shown on Figure 10. The contraction of a polymer chain can be defined by the ratio:

$$
\frac{L_{E}}{L_{C}}=\frac{D P n \times l}{\sqrt{\left\langle h^{2}\right\rangle}}(8)
$$

Where $D P n \times l$ corresponds to the contour length of the polymer chains in the extended state and $\left\langle h^{2}\right\rangle$ corresponds to the average end-to-end distance of polymer chains in the contracted state.

In the case of a flexible carbon polymer chain, $\left\langle h^{2}\right\rangle=2 \times D P n \times l^{2}$ giving $L_{E} / L_{C}=D P n /$ $\sqrt{2 \times D P n}$. In the case of molecularly dispersed cellulose chains, the contraction can be estimated from the radius of gyration $R g$ and molar mass $M w$ data measured in the solvent Cd-tren by Saalwchter et al. (2000) and Schulz et al. (2000). The contraction of cellulose chains from various sources (bacterial celluloses, cotton linters and wood pulps) in Cd-tren is then calculated by using equation (8) with the following parameters: $D P n=\left(\mathrm{Mw} / 162 \mathrm{~g} . \mathrm{mol}^{-1}\right)$ $/(M w / M n)$ considering a ratio $M w / M n \approx 2$ as assumed by Saalwchter et al. $(2000) ; l \approx 5.15 \AA$ (length of one anhydroglucose unit in the cellulose crystal lattice) $\left\langle h^{2}\right\rangle \approx 6 \times R g^{2}$ corresponds to the average end-to-end distance of cellulose chains in the contracted state.

As can be seen on Figure 10, the contraction increases very fast as a function of $D P n$ for a flexible polymer chain under vacuum. At a low $D P n$ value of 400 , the contraction ratio reaches 14. In the case of cellulose chains in Cd-tren, the contraction is lower due to the higher chain stiffness caused by the extensive hydrogen bonds network occurring within and between cellulose chains. As demonstrated by Saalwchter et al. (2000) and Schulz et al. (2000), molecularly dissolved celluloses behave as semi flexible polymer chains. Consequently, the calculated contraction ratios are 4.6 to 5.1 times lower for bacterial celluloses and cotton linters and 5.4 to 5.9 times lower for wood pulps as compared to flexible polymer chains. Besides, the interaction polymer - solvent, i.e. cellulose / Cd-tren, plays obviously a role on the average end-to-end distance and thus the degree of contraction of the chains. The contraction measured for cellulose fibers in NMMO - 20 to $23 \%$ water w/w, are giving much lower values especially for high $D P n$ values. This is clearly due to the fact that there is a large difference between the contraction of individual chains and the one of the whole fiber, at least for three reasons. Firstly, the swollen fiber is not fully dissolved (crystalline regions are less affected than amorphous ones), which means that not all chains are participating to the contraction. Secondly, the solvent is not individualizing all chains, and 
the presence of chain aggregates decreases the contraction possibilities. Finally, there should be a strong non-linearity of the process due to the fact that the cooperativity between adjacent chains may be low due to hydrogen bond breakage.

\section{Conclusions}

The rotation and contraction movements that occur upon swelling and dissolution of cellulose fibers are strongly linked to the morphological structure of the cellulose fibers. The "microscopic rotation" and the rolling up of the primary wall, occur only for native fibers as cotton or wood. The rolling up of the primary wall originates from stress unbalances due to the change of orientation and extension of the chains during the cell elongation phase of the growth. Contraction upon swelling is seen for both native and regenerated fibers. It originates from the fact that cellulose chains are in an extended conformational state due to the spinning process for the regenerated cellulose fibers or to the bio-deposition process for native cellulose fibers. The contraction is related to the change of the mean conformation of cellulose chains from an extended state to a more condensed state.

Acknowledgements. The authors thank Borregaard, Dow Wolff Cellulosics GmbH, Lenzing AG and Spontex for their financial and technical support and their staff for scientific discussions. They thank INRA Versailles for providing cotton samples.

\section{References}

Abu-Rous M, Ingolic E, Schuster KC (2006) Visualisation of the fibrillar and pore morphology of cellulosic fibres applying transmission electron microscopy. Cellulose 4:411-419

Baaijens FTP (1991) Calculation of residual stresses in injection moulded products. Rheol Acta 30:284-299

Brändström J, Bardage SL, Daniel G, Nilsson T (2003) The structural organisation of the S1 cell wall layer of Norway spruce tracheids. IAWA J 24:27-40

Bowling AJ, Amano Y, Lindstrom R, Brown RM Jr (2001) Rotation of cellulose ribbons during degradation with fungal cellulose. Cellulose 8:91-97

Carra JH, Tripp VW, Orr RS (1962) Yarn untwisting as a rapid test of cotton swelling in various reagents. Text Res J 32:1041-1042

Chanzy H, Noe P, Paillet M, Smith P (1983) Swelling and dissolution of cellulose in amine oxide / water systems. J Appl Polym Sci 37:239-259

Chaudemanche C, Navard P (2009) Influence of fibre morphology on the swelling and dissolution mechanisms of Lyocell regenerated cellulose fibres. submitted to Cellulose

Cuissinat C (2006) Swelling and dissolution mechanisms of native cellulose fibres. Thèse de doctorat, Ecole Nationale Supérieure des Mines de Paris, Sophia-Antipolis (France) 
Cuissinat C, Navard P (2006a) Swelling and dissolution of cellulose, Part I: free floating cotton and wood fibres in $N$-methylmorpholine- $N$-oxide - water mixtures. Macromol Symp 244:1-18

Cuissinat C, Navard P (2006b) Swelling and dissolution of cellulose, Part II: free floating cotton and wood fibres in $\mathrm{NaOH}$ water-additives systems. Macromol Symp 244:19-30

Cuissinat C, Navard P (2008) Swelling and dissolution of cellulose, Part III: Plant fibres in aqueous systems. Cellulose 15:67-74

Cuissinat C, Navard P, Heinze T (2008a) Swelling and dissolution of cellulose, Part IV: Free floating cotton and wood fibres in ionic liquids. Carbohydr Polym 72:590-596

Cuissinat C, Navard P, Heinze T (2008b) Swelling and dissolution of cellulose, Part V: Cellulose derivatives fibres in aqueous systems and ionic liquids. Cellulose 15:75-80

Diddens I, Murphy B, Krisch M, Müller M (2008) Anisotropic elastic properties of cellulose measured using inelastic X-ray scattering. Macromolecules 41:9755-9759

El-Shiekh A, Bogdan JF, Gupta RK (1971) The Mechanics of bicomponent fibers: Part I : theoretical analysis. Text Res J 41:281-297

Flemming N, Thaysen AC (1919) On the deterioration of cotton on wet storage. Biochem J 14:25-29

Flemming N, Thaysen AC (1921) On the deterioration of cotton on wet storage. Biochem. J. 14:407-415

Giroud T, Vincent M (2004) Evaluation of residual stresses in short fiber reinforced thermoplastic molded parts. Mec Ind 5:481-487

Hensen F (1989) New developments in fiber and film extrusion. Intern Polym Process 4:64-72

Hock CW (1950) Degradation of cellulose as revealed microscopically. Text Res J 20:141-151

Hock CW (1954) Structures and properties of cellulose fibers - microscopic structure. In: Ott E, Spurlin HM, Grafflin MW (eds) Cellulose and cellulose derivatives (Part 1), 2nd edn. Interscience Publisher, New York, London, pp 347-392

Hsieh Y-L (1999) Structural development of cotton fibers and linkages to fiber quality. In: Basra AS (ed) Cotton fibers: developmental biology, quality improvement and textile processing. Food Products Press/Haworth Press, New York, London, Oxford, pp 137-165

Isayev AI, Kwon K (2009) Volumetric and anisotropic shrinkage in injection moldings of thermoplastics. In Kamal MR, Isayev AI, Liu S-J (eds) Injection molding - technology and fundamentals. Hanser, Munich, pp $779-807$

Jianchin Z, Meiwu S, Zhu H, Kan L (1999) Study of the skin-core structure of lyocell staple fibers. Chem Fibers Int 49:496-500

Kassenbeck P (1970) Bilateral structure of cotton fibers as revealed by enzymatic degradation. Text Res J 40:330-334

Klemm D, Philipp B, Heinze T, Heinze U, Wagenknecht W (1998) General considerations on structure and reactivity of cellulose. In: Comprehensive cellulose chemistry. Vol. 1, Wiley-VCH, Weinheim, pp 9-165

Klemm D, Schmauder HP, Heinze T (2002) Cellulose. In: Vandamme EJ, De Baets S, Steinbüchel A (eds) Biopolymers, Vol. 6. Wiley-VCH, Weinheim, pp 275-319

Krässig HA (1993) Cellulose - Structure, Accessibility and Reactivity. Polymer Monographs, Vol. 11. Gordon and Breach Science Publishers, Amsterdam

Le Moigne N, Montes E, Pannetier C, Höfte H, Navard P (2008) Gradient in dissolution capacity of successively deposited cell wall layers in cotton fibres. Macromol Symp 262:65-71 
Le Moigne N, Spinu M, Heinze T, Navard P (2009) Restricted dissolution and derivatization capacities of cellulose fibers under uniaxial elongational stress. accepted for publication in Polymer

Lenz J, Schurz J, Wrentschur E (1993) Properties and structure of solvent-spun and viscose-type fibres in the swollen state. Colloid Polym Sci 271:460-468

Matsuo T (1977) Polypropylene fibers crimped by asymmetrical quenching. J Text Mach Soc Japan 23:29-34

Nägeli C (1864) Über den inneren Bau der vegetabilischen Zellmembranen. Sitzber Bay Akad Wiss München $1: 282-323,2: 114-171$

O'Sullivan AC (1997) Cellulose: the structure slowly unravels. Cellulose 4:173-207

Paredez AR, Somerville CR, Ehrhardt DW (2006) Visualization of Cellulose Synthase Demonstrates Functional Association with Microtubules. Science 312:1491-1495

Paris A (1992) Influence des conditions de filage sur les propriétés physiques et mécaniques de fibres de polypropylène. Thèse de doctorat, Ecole Nationale Supérieure des Mines de Paris, Sophia-Antipolis (France)

Pennetier G (1883) Note micrographique sur les altérations du cotton. Bull Soc Ind Rouen 11:235-237

Roelofsen PA (1959) The cell-wall structure. In: The plant cell-wall. Gebrüder Borntraeger, Berlin, pp 102-305

Rollins ML, Tripp VW (1954) Optical and electron microscopic studies of cotton fiber structure. Text Res J $24: 345-357$

Ryser U (1999) Cotton fiber initiation and histodifferentiation. In: Basra AS (ed) Cotton fibers: developmental biology, quality improvement and textile processing. Food Products Press/Haworth Press, New York, pp 145

Saalwchter K, Burchard W, Klfers P, Kettenbach G, Mayer P, Klemm D, Dugarmaa S (2000) Cellulose solutions in water containing metal complexes. Macromolecules 33:4094-4107

Sahlberg U, Salmén L, Oscarsson A (1997) The fibrillar orientation in the S2-layer of wood fibers as determined by X-ray diffraction analysis. Wood Sci Technol 31:77-86

Schurz, J. (1994) Was ist neu an den neuen Fasern der Gattung Lyocell ? Lenzinger Ber 74:37-40

Schulz L, Seger B, Burchard W (2000) Structures of cellulose in solution. Macromol Chem Phys 201:2008-2022

Treuting RG, Read WT (1951) A mechanical determination of biaxial residual stress in sheet materials. J Appl Phys 22:130-134

Tripp VW, Rollins ML (1952) Morphology and chemical composition of certain components of cotton fiber cell wall. Anal Chem 24:1721-1728

Warwicker JO, Jeffries R, Colbran RL, Robinson RN (1966) A review of the literature on the effect of caustic soda and other swelling agents on the fine structure of cotton. Shirley Institute Pamphlet No. 93, St Ann's Press, England 


\section{Figure legends}

Fig. 1 (a) First step: access of the solvent inside the fiber through the primary wall; (b) Second step: excrescence of the balloons, swelling of the S1 wall due to the dissolution of the S2 wall, breakage and rolling up of the primary wall forming collars and threads; (c) Third step: shaping of the balloons, the S2 wall is fully dissolved and hold by the S1 wall which forms the membrane of the balloons. The primary wall that formed threads and collars surrounds the balloons.

Fig. 2 Calculated unrolled length $L$ of the two cylinders as a function their radius as compared to the initial perimeter of cellulose fibers ranging from 10 to $30 \mu \mathrm{m}$ in diameter.

Fig. 3 (a) Macroscopic rolling up of the primary wall associated to the sum of the bending of each representative elementary volume, and definition of the $x, y$ and $z$ axes. (b) Normal forces along the $(x)$ and $(y)$ directions for a representative elementary volume.

Fig. 4 Twists of a dried cotton hair observed by scanning electron microscopy.

Fig. 5 Formation of helices for a cotton hair, due to the "microscopic rotation" that occurs during the swelling and dissolution.

Fig. 6 "Microscopic rotation" for cotton hairs as revealed by the orientation of the microfibrils in the swollen membrane. "(a) The fiber is attached at its two ends and the "microscopic rotation" cannot occur. The microfibril orientation, difficult to be seen, is visible as pale white lines (black arrow). It almost keep its initial helical orientation. (b) The fiber is freely floating in the solvent and swells by ballooning. The microscopic rotation unwinds the initial organization seen on fig 6a. The microfibrils (black arrow) are now aligning along the fiber axis.

Fig. 7 (a) Helices in the case of a BBUE wood pulp fiber. The threads stay at the edge of the balloons (white arrows); (b) Large homogeneous swelling in the case of a regenerated cellulose fiber. Due to the large swelling, the optical contrast between the swollen fiber and the outside medium is very low and it is difficult to see the fiber. The two arrows are positioned at its two sides. There is no detachment of any part, and thus no threads, helices and collars. 
Fig. 8 Formation of helices or rings without "microscopic rotation" due to the breakage of the primary wall at two diametrically opposite points.

Fig. 9 (From a to b) Contraction during the swelling and dissolution of a VHV wood pulp fiber.

Fig. 10 Contraction ratio vs $D P n$ calculated for a flexible polymer chain and for cellulose chains from different sources (bacterial celluloses, cotton linters, wood pulps) molecularly dispersed in Cd-tren ( $R g$ and $M w$ data taken from Saalwchter et al. 2000; Schulz et al. 2000) as compared to the contraction measured for regenerated fibers, bleached cotton hairs and VHV wood pulp fibers. 

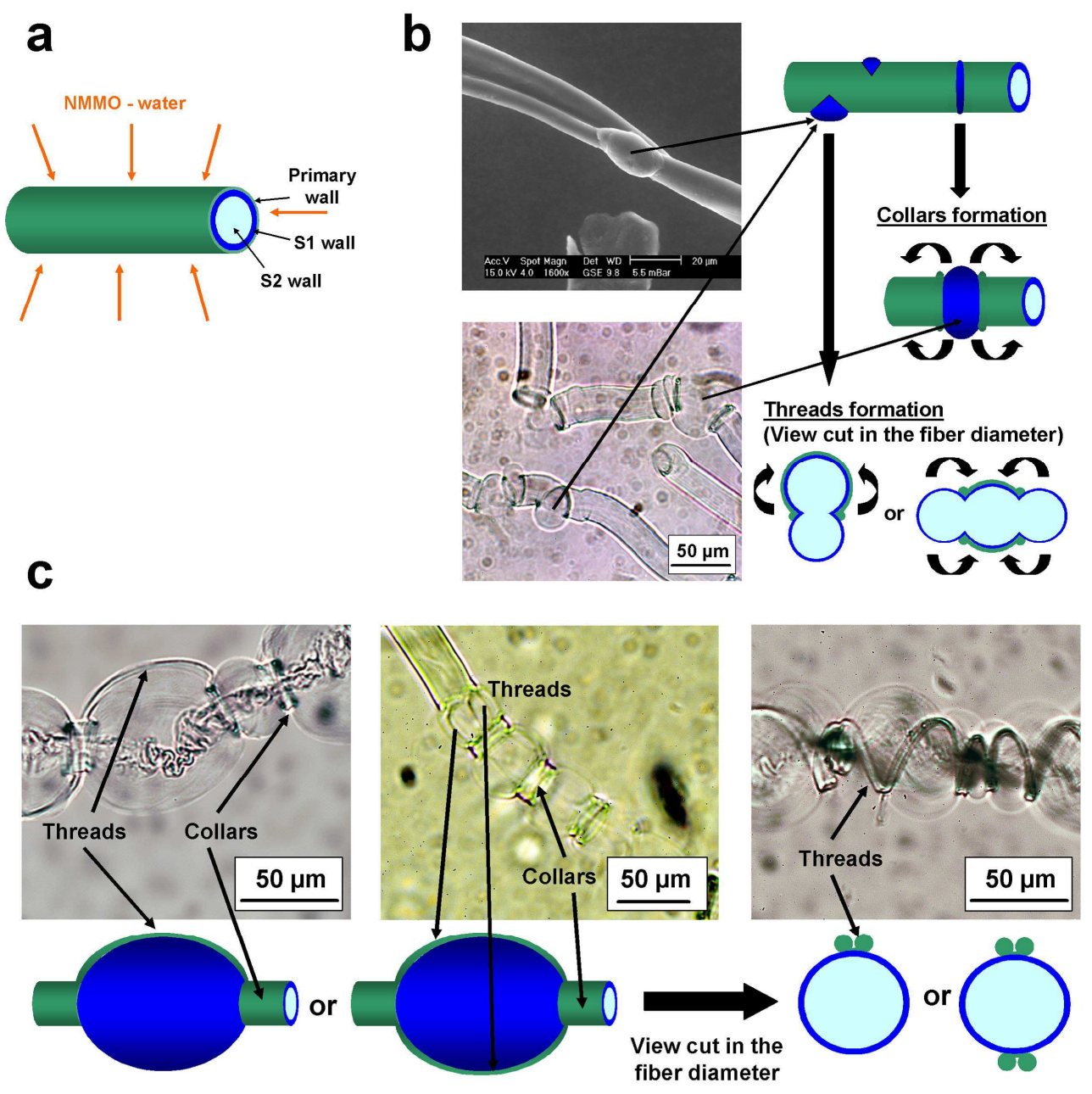

Fig. 1 


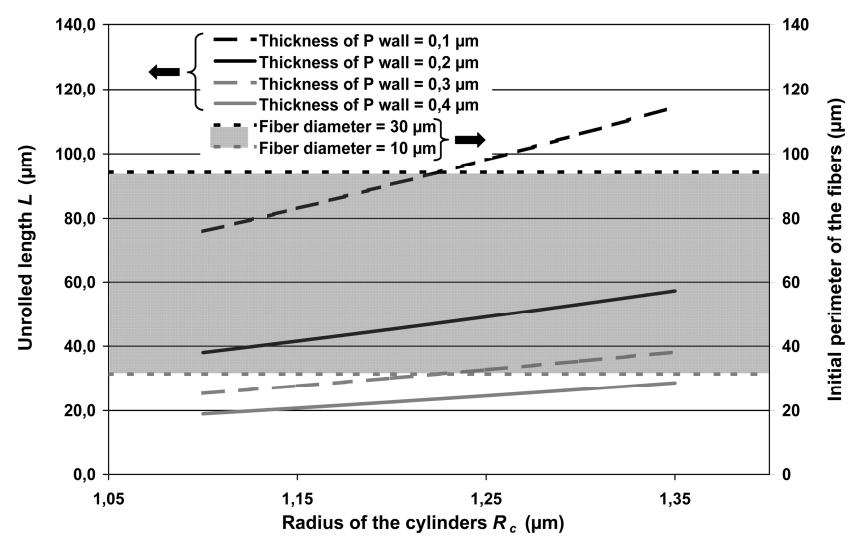

Fig. 2 


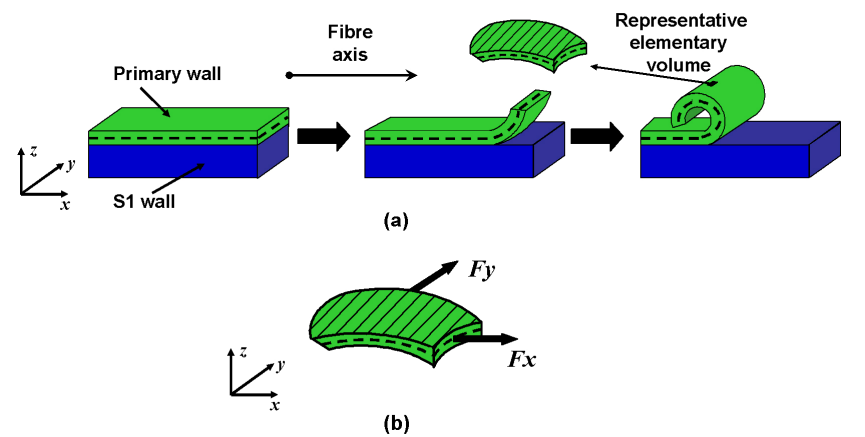

Fig. 3 


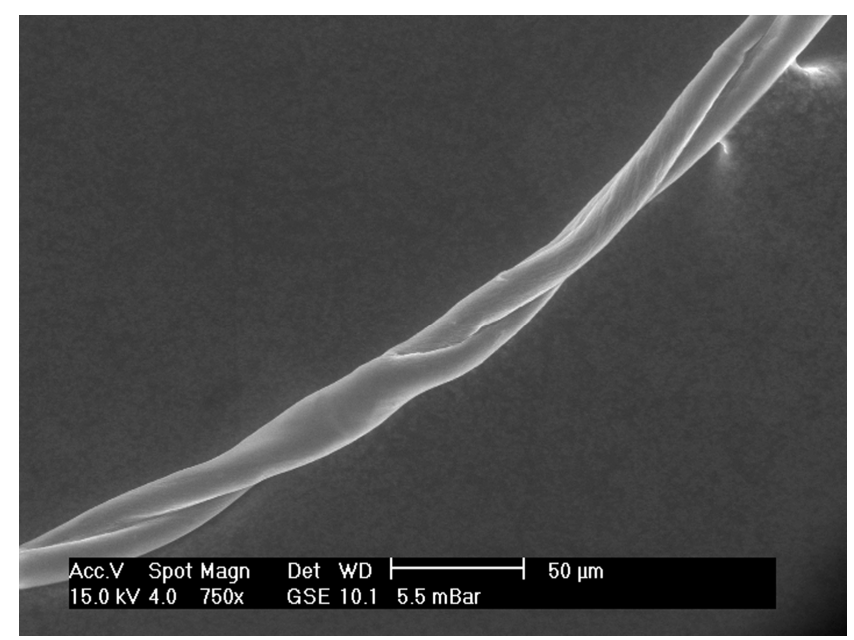

Fig. 4 


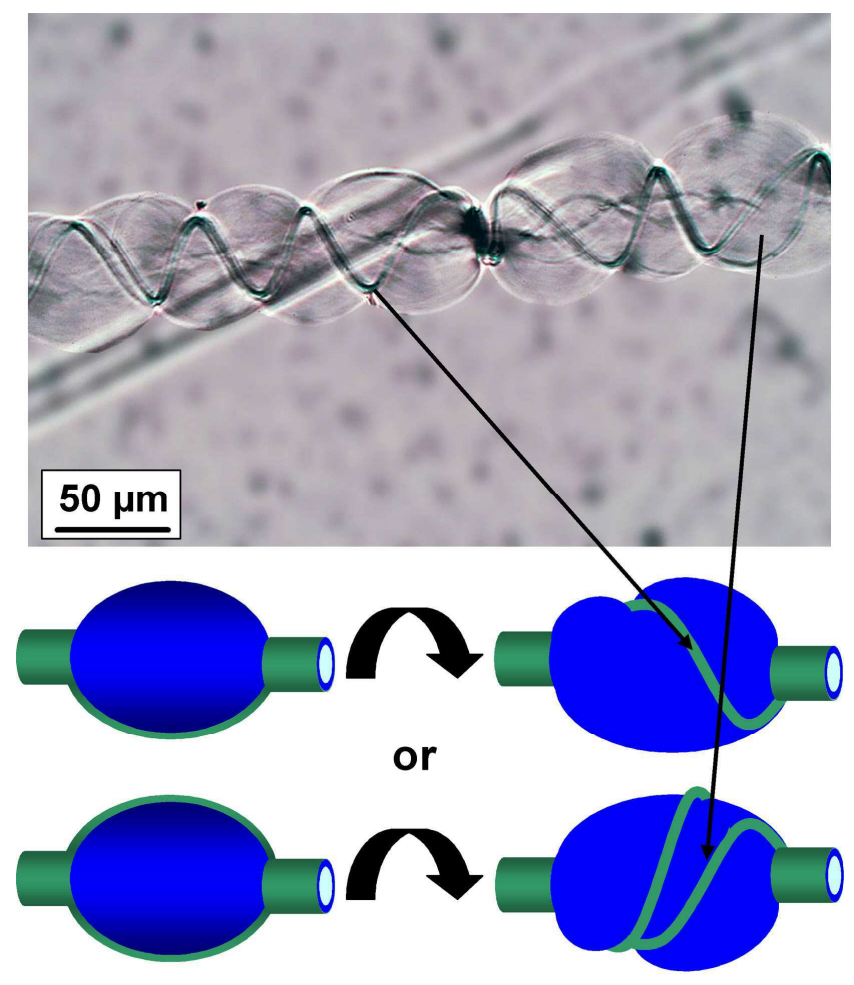

Fig. 5 

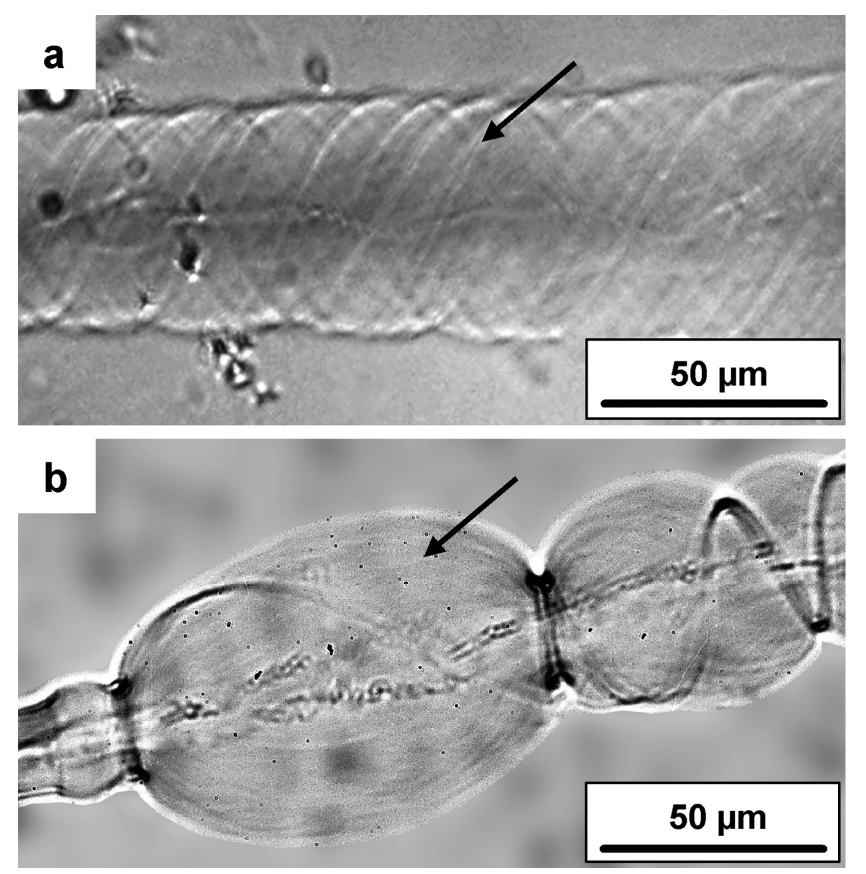

Fig. 6 


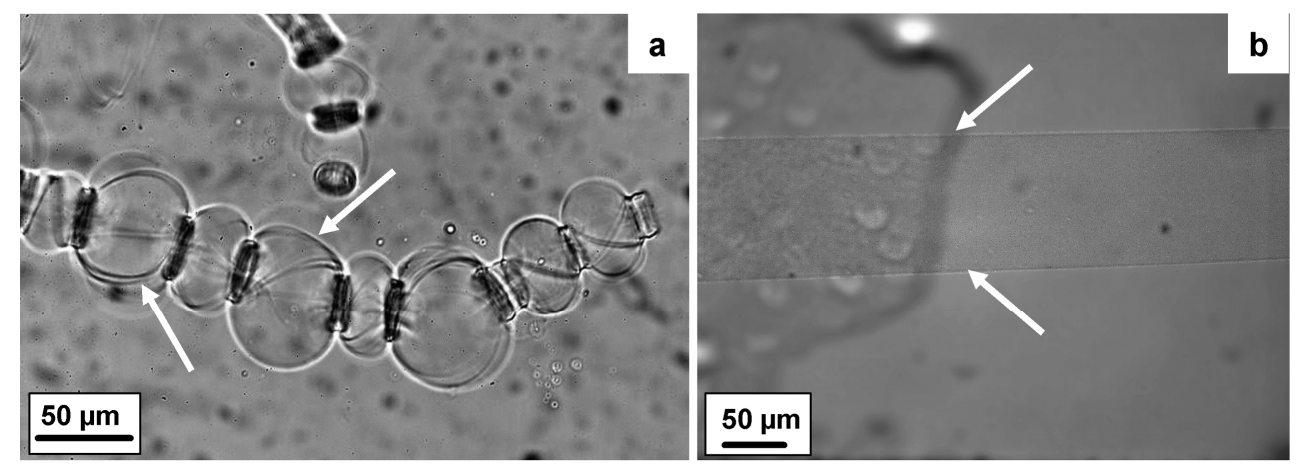

Fig. 7 


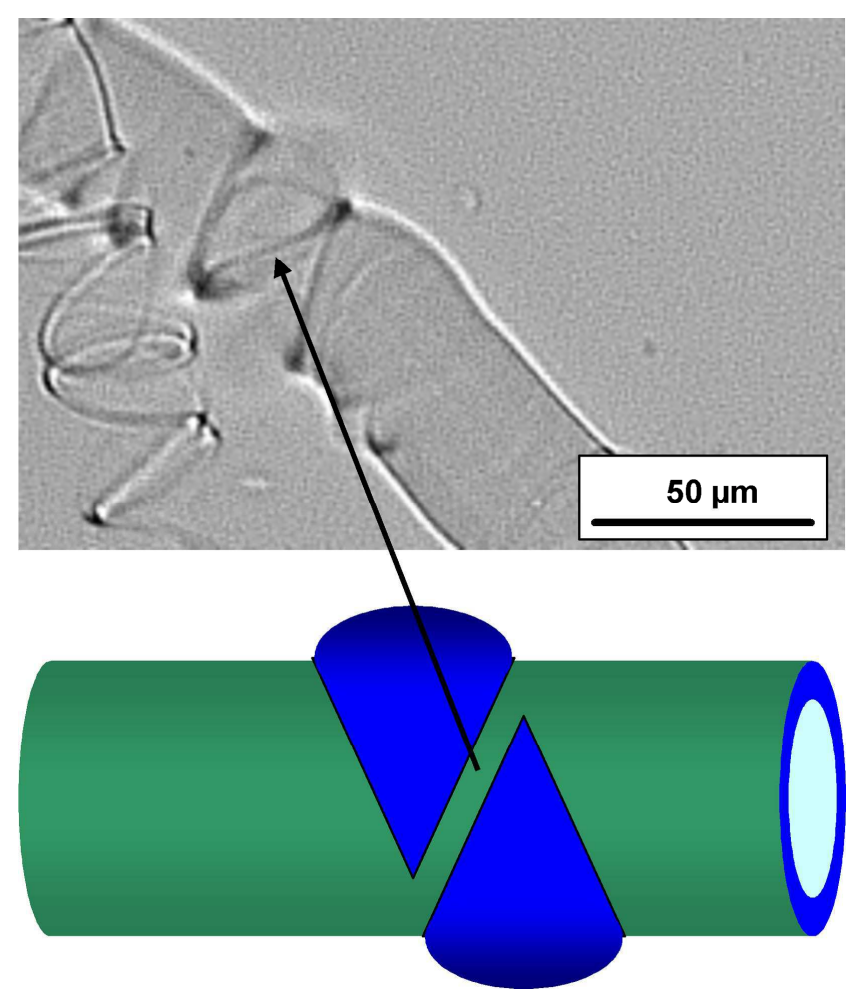

Fig. 8 


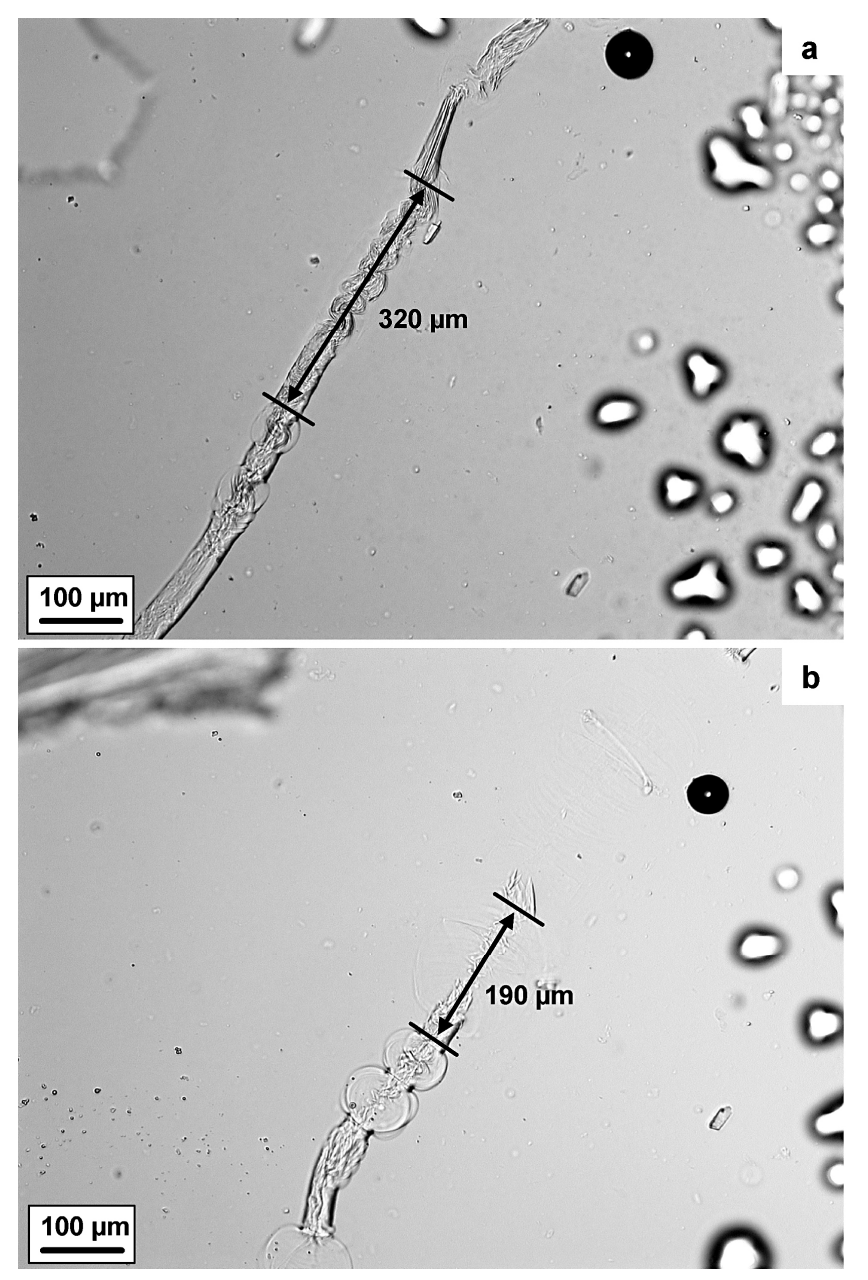

Fig. 9 


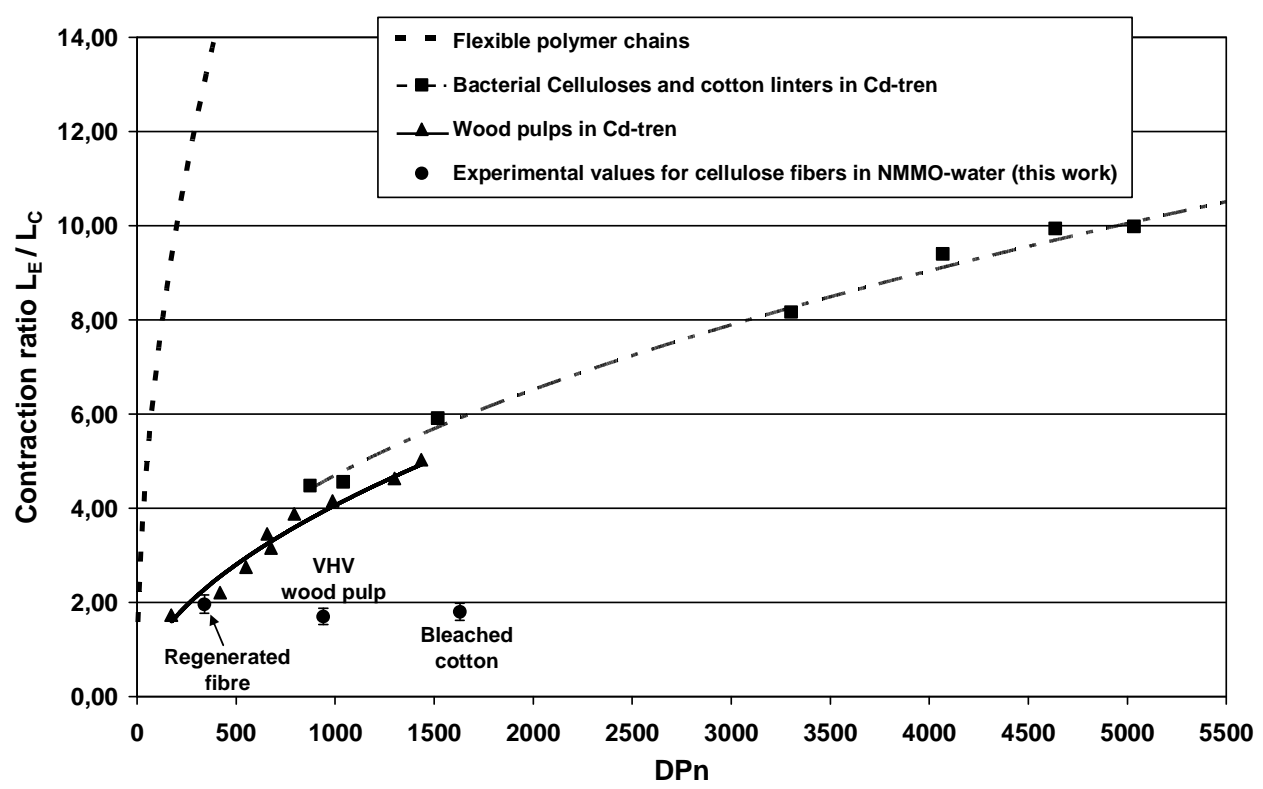

Fig. 10 